\title{
Personalisation of Cellular Electrophysiology Models: Utopia?
}

\author{
Michael Clerx \\ Computational Biology \& Health Informatics, \\ Department of Computer Science, University of Oxford, UK
}

\begin{abstract}
As cell-level differences from person to person are gaining more attention, the idea of having personalised models of cell electrophysiology is growing ever more attractive. In this paper for the special session "Personalized medicine through integration of imaging and cardiac modeling”, I briefly review the different pathways to personalisation and the challenges they present.
\end{abstract}

\section{Introduction}

Detailed models of the cardiomyocyte (CM) action potential (AP) have been used as the basis for multi-scale investigations into the healthy heart, arrhythmias, cardiomyopathies, and the effects of drugs or genetic mutations. While initially AP models were used exclusively to test mechanistic hypotheses or study difficult-to-measure variables, they are now used increasingly for prediction, e.g. in safety pharmacology [1] or clinical risk assessment in patients with genetic mutations [2]. At the same time, it has become increasingly accepted that person-to-person differences manifest even at the level of cell electrophysiology, and may need to be taken into account when modelling disease mechanisms [3]. If AP models could be personalised, mechanistic modelling studies could be repeated for individual patients and form the basis for diagnosis and tailored treatment, in the same way that personalised macroscopic models are used already [4,5].

Figure 1 shows the different roads to personalisation. Conceptually, the most direct method is shown at the bottom, simply take all the cells of interest and perform the experiments needed to characterise them completely. The practical and ethical difficulties associated with this route give rise to the more complicated clouds of tailoring options shown above, as discussed in the remainder of this paper.

\section{Species-specific models: utopia?}

Human-specific models are an obvious starting point for patient-specific models. But how much of their data is human, and how do we go about replacing it with fresh patient-specific data? Analyses such as $[1,6,7]$ partially address the first question, and show that AP models are almost exclusively multi-species — in fact, while Hodgkin and Huxley's model was entirely squid, the first cardiac AP model already mixed species [8] and this has continued largely unchanged, the only exception perhaps being the rabbit model by Gray et al. [9]. A secondary (and solvable) problem that these analyses highlight is that AP model history is so complex that tracing the origins of model parameters is a challenging and time-consuming task. A partial solution to this problem, suggested in [10], is to use tools such as CellML [11] and the Physiome Model Repository [12] to build a database of models and annotate the origins of equations and parameters, building a browsable network of model phylogeny. This would provide modellers with a weak description of model provenance; not fully explaining how a model was created but at least pointing to the relevant literature. A stronger provenance description could be made by formalising and storing model fitting procedures, with complete reference to the used algorithms and data sets. This is the aim of the Cardiac Electrophysiology Web Lab project $[10,13]$, which would provide modellers with the information (and code) needed to completely reparametrise a model to patient-specific data.

Given an AP model, which parts can and should we personalise? At first glance, it seems a model's parameter values describe the quantities that can be measured and vary from person to person, while its equations reflect the shared physiological mechanisms, and so will not change. However, there are exceptions: the 1795insD mutation in the sodium channel gene $S C N 5 A$ has been described using an extended Markov model formulation of $\mathrm{I}_{\mathrm{Na}}$, containing states not found in the wild-type model [14].

If we do restrict ourselves to parameter values, there are still parts that may be off-limits. For example, despite nearly 40 years of modelling there is still no consensus model or model structure for calcium dynamics, making it unlikely that calcium handling will be patient-specific soon. Similarly, the experimental difficulty of measuring small transporter or pump currents - key players in restoring ionic concentration gradients [7] — makes them under 

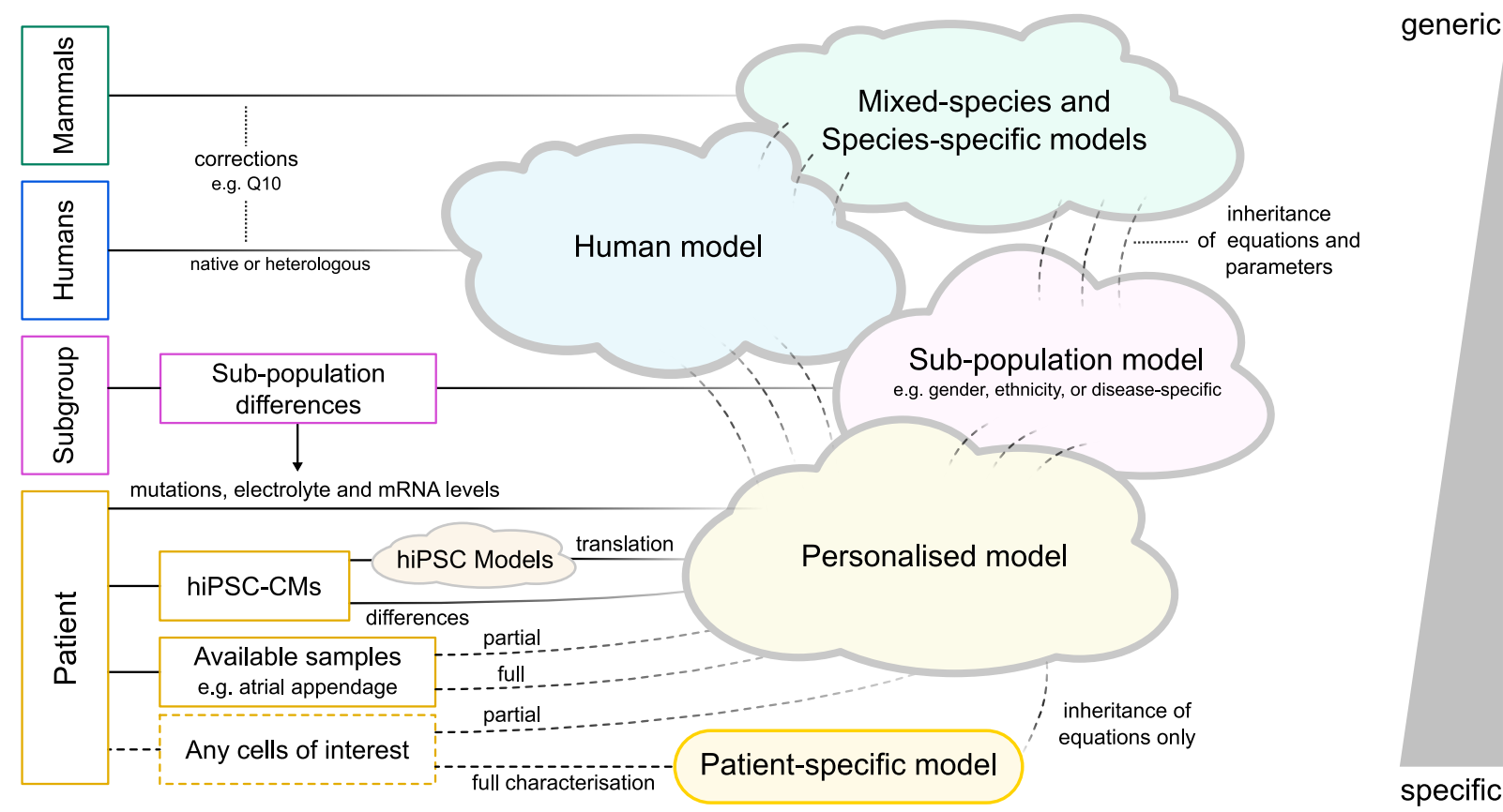

Figure 1. Roads to patient-specific action potential (AP) models. Data from several subjects from several species, collected under varying circumstances, feed into a collection of myocyte models that then share and re-use equations and parameter values between them. By selecting a sub-population closer to the patient, these population data can be used to create a first line of tailored models. Further customisation is possible using minimally-invasive measurements (i.e. blood samples) to incorporate notable differences such as electrolyte imbalances or strong under/over-expression of channel genes. A different route is to use patient-derived cardiomyocyte-like stem cells (hiPSC-CMs), although this requires translation to the adult $\mathrm{CM}$ context (possibly using computational models). In some cases, myocytes can be obtained directly from patients undergoing an operation - but with the caveat that these might not be from the region we're most interested in, and may be affected by other disease mechanisms than the ones we're trying to study. Because the success-rate of experiments on such human samples is low, only a small part of their behaviour can be characterised, which is shown as "partial characterisation" in the figure. Finally, a hypothetical scenario is shown where all desired cell types can be fully (and non-invasively) characterised, leading to completely patient-specific models.

represented in the experimental and modelling literature and unlikely targets for personalisation.

Turning our attention to ion channels, we can make a distinction between maximal conductance parameters and the parameters describing current kinetics. A current's maximal conductance correlates with the number of channels in the membrane, and varies over time as channels degrade and are replaced (e.g. $\mathrm{I}_{\mathrm{Na}}$ and $\mathrm{I}_{\mathrm{Kr}}$ channels have a lifespan of 35 and 10 hours respectively $[15,16])$. This has made conductance parameters a natural target for population and patient-specific models [3,17-19]. Finally, there is some evidence for variability in the kinetics of ionic currents $[20,21]$.

\section{Patient-specific data: utopia?}

A first step towards personalisation is to focus on an appropriate sub-population, e.g. by choosing a model appro- priate to the cell type thought to be the origin of arrhythmic events, or by tailoring a model to a patient's age, ethnicity, or sex [22]. Similarly, models can be made to incorporate cell-level differences known to be associated with the patient's condition (e.g. electrical remodelling).

Some patient, but not heart-specific, data may also be obtained e.g. by partial sequencing, or measuring electrolyte or mRNA levels in blood samples. With care, and possibly the use of added population data, these may be used to include mutation effects, altered ionic concentrations, and coarse changes in ionic current densities.

In some cases, cardiac tissue samples may even be available, for example from atrial appendages. However, obtaining more than one or two measurements from a single patient is rare, so that these data have not yet been used for personalisation. The expertise required for these procedures is rarely found in a single centre, adding to the practical difficulties of this route. 
Even in cases where human data are available, the experimental difficulty may necessitate measuring under nonphysiological conditions and subsequent correction of the measured values. Uncertainty in these corrections could overshadow patient-specific differences one hoped to apply (e.g. compare the $30 \mathrm{mV}$ corrections to human $\mathrm{I}_{\mathrm{Na}}$ data employed in [23] to the mean voltage shift of around $5 \mathrm{mV}$ caused by $\mathrm{I}_{\mathrm{Na}}$ mutations [24]).

A final way of obtaining patient-specific data is to perform electrophysiological measurements in patientderived hiPSC-CMs. Genetically, hiPSC-CMs are an ideal model for the patient's myocytes, but it remains to be determined how much of a myocyte's behaviour is genetic, and how much is defined by its environment and history. While early investigations showed marked differences between hiPSC-CM and adult CM electrophysiology [25], many advances in the hiPSC maturation process have since been made (e.g. [26]).

If we accept that hiPSC-CM electrophysiology does differ from that of native myocytes, the question arises how to translate results in hiPSC-CMs to the adult CM context. One method that has been used is to measure stem cells derived from both the patient and a sibling (e.g. one without a particular ion channel mutation), and then inspect the differences [27]. A second option would be to investigate a proarrhythmic mechanism (e.g. the formation of an EAD) in the stem-cell context, using stem-cell measurements and a model of the stem-cell AP [28, 29]. This mechanism could then be recreated in a native myocyte model, by manual tuning of its parameters. Whether or not the advantages of these methods outweigh the added cost compared to e.g. heterologous expression experiments remains an open question.

\section{Cell-specific models: utopia?}

Given both a good baseline model and patient-specific tissue samples, can we create patient or even cell-specific models? Typical experiments focus on a single aspect, e.g. a single current, and then use averaged data from several cells for analysis. However, studies have shown that at least to some extent, it is possible to measure multiple currents in a single CM [30]. Similarly, multiple maximum conductances can be estimated from recordings in a single cell [18,31], including hiPSC-CM [32]. These studies, however, start from the assumption of perfect knowledge of the ionic current dynamics, and point to this as an area where their methodology can be improved. Rapid methods to characterise ion current dynamics have recently been developed $[21,33,34]$ and show promising results. However, a study has yet to appear that attempts this approach for more than one current, or extends it to other areas such as calcium handling or pumps and exchangers.

\section{Conclusion}

Arguably, personalised cell models have been around at least since 1999, when Clancy and Rudy incorporated a patient-specific mutation into an AP model [14]. But a fully personalised model, where every parameter is patient-derived, still seems a distant dream. Interestingly, many of the challenges encountered trying to move forward in this way uncover unsolved problems from the past: Do we understand native CMs well-enough that we can translate our novel stem-cell findings to them? Have we been careful enough in documenting the data sources and fitting procedures for our models? And are we learning enough, and quickly enough, from our routine experiments? These fundamental challenges will need to be addressed to make personalised cell modelling a reality.

Acknowledgements - I would like to thank Jordi Heijman and Gary Mirams for our valuable discussions. All opinions expressed in the text are my own.

\section{References}

[1] Davies MR, Wang K, Mirams GR, Caruso A, Noble D, Walz A, Lavé T, Schuler F, Singer T, Polonchuk L. Recent developments in using mechanistic cardiac modelling for drug safety evaluation. Drug Discovery Today 2016; 21(6):924-938.

[2] Hoefen R, Reumann M, Goldenberg I, Moss AJ, Jin O, Gu Y, McNitt S, Zareba W, Jons C, Kanters JK, et al. In silico cardiac risk assessment in patients with long QT syndrome: type 1: clinical predictability of cardiac models. Journal of the American College of Cardiology 2012;60(21):21822191.

[3] Ni H, Morotti S, Grandi E. A heart for diversity: simulating variability in cardiac arrhythmia research. Frontiers in Physiology 2018;9.

[4] Arevalo HJ, Vadakkumpadan F, Guallar E, Jebb A, Malamas P, Wu KC, Trayanova NA. Arrhythmia risk stratification of patients after myocardial infarction using personalized heart models. Nature Communications 2016;7.

[5] Gray RA, Pathmanathan P. Patient-specific cardiovascular computational modeling: diversity of personalization and challenges. Journal of Cardiovascular Translational Research 2018;1-9.

[6] Niederer S, Fink M, Noble D, Smith N. A meta-analysis of cardiac electrophysiology computational models. Experimental Physiology 2009;94(5):486-495.

[7] Bueno-Orovio A, Sánchez C, Pueyo E, Rodriguez B. Na/K pump regulation of cardiac repolarization: insights from a systems biology approach. Pflugers Archiv European Journal of Physiology 2014;466(2):183-193.

[8] Noble D. A modification of the Hodgkin-Huxley equations applicable to Purkinje fibre action and pacemaker potentials. The Journal of Physiology 1962;160(2):317-352.

[9] Gray RA, Pathmanathan P. A parsimonious model of the 
rabbit action potential elucidates the minimal physiological requirements for alternans and spiral wave breakup. PLoS Computational Biology 2016;12(10):e1005087.

[10] Daly AC, Clerx M, Beattie KA, Cooper J, Gavaghan DJ, Mirams GR. Reproducible model development in the cardiac electrophysiology web lab. Progress in Biophysics and Molecular Biology 2018;

[11] Hedley WJ, Nelson MR, Bellivant D, Nielsen PF. A short introduction to CellML. Philosophical Transactions of the Royal Society of London A Mathematical Physical and Engineering Sciences 2001;359(1783):1073-1089.

[12] Yu T, Lloyd CM, Nickerson DP, Cooling MT, Miller AK, Garny A, Terkildsen JR, Lawson J, Britten RD, Hunter PJ, et al. The Physiome model repository 2. Bioinformatics 2011;27(5):743-744.

[13] Cooper J, Scharm M, Mirams GR. The cardiac electrophysiology web lab. Biophysical Journal 2016;110:292-300.

[14] Clancy CE, Rudy Y. Linking a genetic defect to its cellular phenotype in a cardiac arrhythmia. Nature 1999; 400(6744):566-569.

[15] Maltsev VA, Kyle JW, Mishra S, Undrovinas A. Molecular identity of the late sodium current in adult dog cardiomyocytes identified by Nav1.5 antisense inhibition. American Journal of Physiology Heart and Circulatory Physiology 2008;295(2):H667-H676.

[16] Vandenberg JI, Perry MD, Perrin MJ, Mann SA, Ke Y, Hill AP. hERG K+ channels: structure, function, and clinical significance. Physiological Reviews 2012;92(3):13931478.

[17] Sarkar AX, Christini DJ, Sobie EA. Exploiting mathematical models to illuminate electrophysiological variability between individuals. The Journal of Physiology 2012; 590(11):2555-2567.

[18] Groenendaal W, Ortega FA, Kherlopian AR, Zygmunt AC, Krogh-Madsen T, Christini DJ. Cell-specific cardiac electrophysiology models. PLoS Computational Biology 2015; 11(4):e1004242.

[19] Muszkiewicz A, Britton OJ, Gemmell P, Passini E, Sánchez C, Zhou X, Carusi A, Quinn TA, Burrage K, BuenoOrovio A, et al. Variability in cardiac electrophysiology: Using experimentally-calibrated populations of models to move beyond the single virtual physiological human paradigm. Progress in Biophysics and Molecular Biology 2016;120(1-3):115-127. ISSN 0079-6107.

[20] Clerx M. Variability in the dynamical properties of cardiac ina. In Multi-scale Modeling and Variability in Cardiac Cellular Electrophysiology. Ph.D. thesis. Maastricht University, 2017; 75-106.

[21] Beattie KA, Hill AP, Bardenet R, Cui Y, Vandenberg JI, Gavaghan DJ, de Boer TP, Mirams GR. Sinusoidal voltage protocols for rapid characterization of ion channel kinetics. The Journal of Physiology 2018;.

[22] Yang PC, Clancy CE. In silico prediction of sex-based differences in human susceptibility to cardiac ventricular tachyarrhythmias. Frontiers in Physiology 2012;3:360.

[23] O’Hara T, Virág L, Varró A, Rudy Y. Simulation of the undiseased human cardiac ventricular action potential: model formulation and experimental validation. PLOS Computational Biology May 2011;7(5):e1002061.

[24] Clerx M, Heijman J, Collins P, Volders PGA. Predicting changes to INa from genetic variants in human SCN5A. Scientific Reports 2018;

[25] Blazeski A, Zhu R, Hunter DW, Weinberg SH, Zambidis ET, Tung L. Cardiomyocytes derived from human induced pluripotent stem cells as models for normal and diseased cardiac electrophysiology and contractility. Progress in Biophysics and Molecular Biology 2012;110(2-3):166177.

[26] Ronaldson-Bouchard K, Ma SP, Yeager K, Chen T, Song L, Sirabella D, Morikawa K, Teles D, Yazawa M, VunjakNovakovic G. Advanced maturation of human cardiac tissue grown from pluripotent stem cells. Nature 2018; 556(7700):239.

[27] Ma D, Wei H, Zhao Y, Lu J, Li G, Sahib NBE, Tan TH, Wong KY, Shim W, Wong P, et al. Modeling type 3 long QT syndrome with cardiomyocytes derived from patientspecific induced pluripotent stem cells. International Journal of Cardiology 2013;168(6):5277-5286.

[28] Paci M, Hyttinen J, Rodriguez B, Severi S. Human induced pluripotent stem cell-derived versus adult cardiomyocytes: an in silico electrophysiological study on effects of ionic current block. British Journal of Pharmacology 2015; 172(21):5147-5160.

[29] Koivumäki JT, Naumenko N, Tuomainen T, Takalo J, Oksanen M, Puttonen KA, Lehtonen Š, Kuusisto J, Laakso M, Koistinaho J, et al. Structural immaturity of human ipscderived cardiomyocytes: In silico investigation of effects on function and disease modeling. Frontiers in Physiology 2018;9:80.

[30] Banyasz T, Horvath B, Jian Z, Izu LT, Chen-Izu Y. Sequential dissection of multiple ionic currents in single cardiac myocytes under action potential-clamp. Journal of Molecular and Cellular Cardiology 2011;50(3):578-581.

[31] Johnstone RH, Chang ET, Bardenet R, De Boer TP, Gavaghan DJ, Pathmanathan P, Clayton RH, Mirams GR. Uncertainty and variability in models of the cardiac action potential: Can we build trustworthy models? Journal of Molecular and Cellular Cardiology 2016;96:49-62.

[32] Lei CL, Wang K, Clerx M, Johnstone RH, HortigonVinagre MP, Zamora V, Allan A, Smith GL, Gavaghan DJ, Mirams GR, et al. Tailoring mathematical models to stemcell derived cardiomyocyte lines can improve predictions of drug-induced changes to their electrophysiology. Frontiers in Physiology 2017;8.

[33] Fink M, Noble D. Markov models for ion channels: versatility versus identifiability and speed. Philosophical Transactions of the Royal Society of London A Mathematical Physical and Engineering Sciences 2009;367(1896):21612179.

[34] Clerx M, Collins P, Volders PGA. Applying novel identification protocols to Markov models of INa. In Computing in Cardiology, volume 42. CINC, 2015; 889-892.

Address for correspondence:

michael.clerx@cs.ox.ac.uk 